\title{
Reproducción del artículo de Juan Carlos Torre, "Una nueva oposición social", originalmente publicado en la revista Los Libros. Un mes de publicaciones en América Latina, n 21, agosto 1971
}

\author{
Maria Cristina Tortti \\ Instituto de Investigaciones en Humanidades y Ciencias Sociales(IdIHCS-CONICET), \\ Facultad de Humanidades y Ciencias de la Educación, Universidad Nacional de La Plata, Argentina \\ mctortti@gmail.com \\ Mora González Canosa \\ Instituto de Investigaciones en Humanidades y Ciencias Sociales(IdIHCS-CONICET), \\ Facultad de Humanidades y Ciencias de la Educación, Universidad Nacional de La Plata, Argentina \\ gonzalezcanosa@yahoo.com.ar
}

A continuación, reproducimos el artículo publicado en agosto de 1971 por Juan Carlos Torre en la revista Los Libros. Un mes de publicaciones en América Latina ${ }^{1}$. Ello nos permite incorporar al dossier un trabajo escrito en la época, la mirada sobre los hechos de Córdoba de una de las publicaciones políticoculturales más importantes del período y el análisis de un reconocido investigador que continuó realizando significativos aportes sobre el tema ${ }^{2}$. En 'Una nueva oposición social”, Torre ve en la protesta del 69' un acontecimiento "abierto" al que, posteriormente, la movilización obrera habría dado un novedoso perfil anticapitalista. Sin embargo, advierte que el devenir de esa ruptura en la cultura obrera -sus posibilidades de expansión- era aún incierto. Llamaba así la atención sobre la asincronía existente entre lo "nuevo" del acontecimiento cordobés y la persistencia, a nivel nacional, de la irresuelta cuestión del peronismo. 


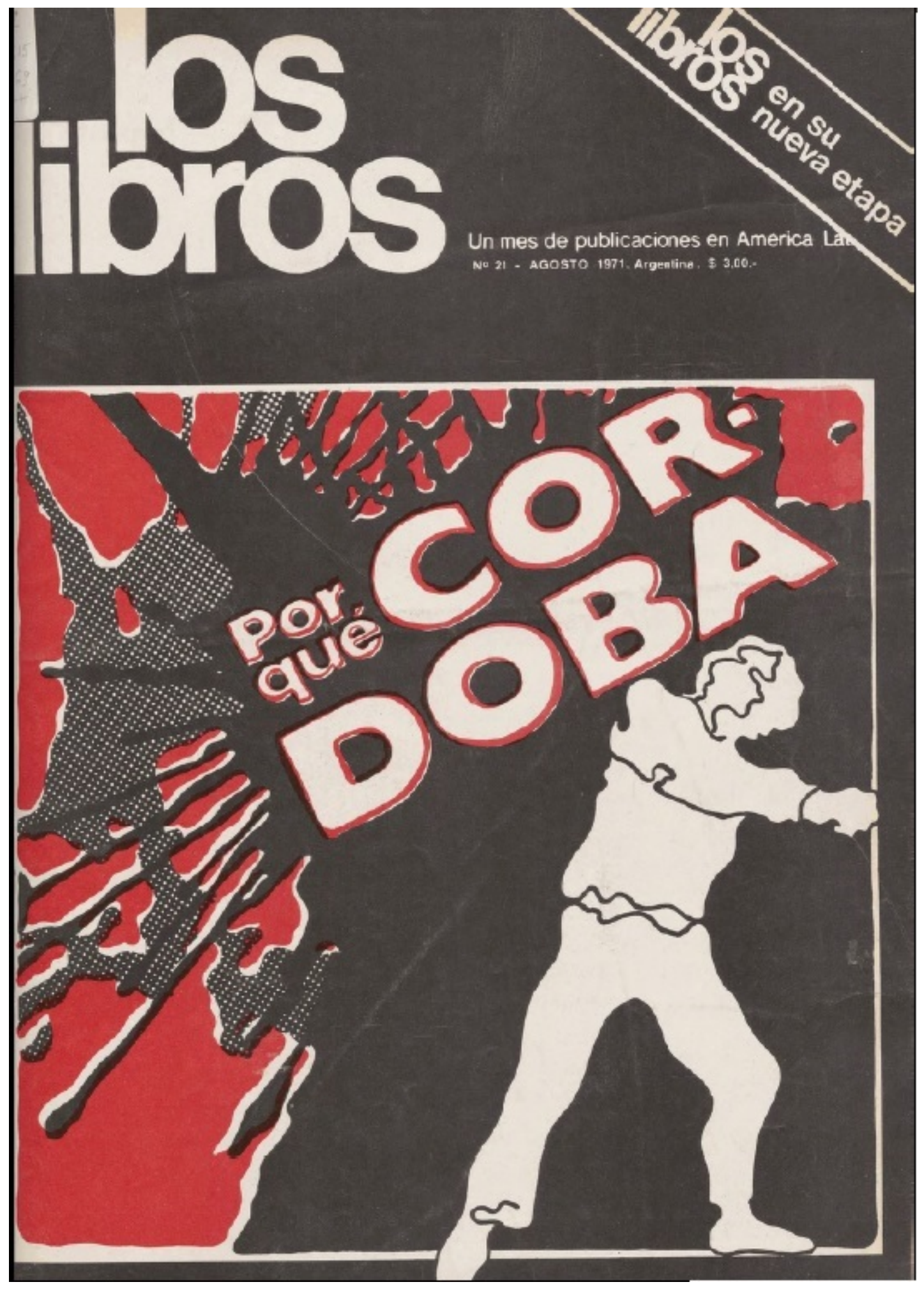




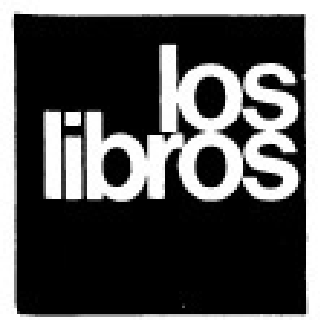

\section{Un mes de publicaciones en América Latina}

AÑO 3 - No 21 - Agosto 1971

\begin{abstract}
Director: Hector Schmucler Secretario:

Correccion: Hayde Valere

Deseho Grifico:

Isabel Carbalio

Corresponales

Chile: Enrique Lihn y Mabet

Piccini; Mkxico: Eligio Calde fon Rodriguez; Venezuela: Adriano Gonzsiez Lebn y Vitmo Vargas: Paraguay: Adolto Fineili: Francia: Silvia Mudni.

LOS LIBROS. Redactiòn $y$ Publicidad: Tuoumán 1427. $z^{\circ}$ p. - of. 207. Tel. 45-9640

\section{Distribuidores:}

ARGENTINA, quiloscos, Buenos Aires, Machi a Cía. S.R.

Libreriass Tres America

Representante para la venta on exterior: Eociciones $M$. certinas, Exportadora Amigos del Libro S.A.t Co lombial Edciones Cruz del Sur: Chile: Editorial Univers! taris'S.A.; Mexico: Antonio Navarrete Ribrefía Hambur90); Paraguay: Selecciones SA.C.; Parú: Distribuidora Garcilaso S.A. Urugesy: Ambrica Latinn: Veneruela: Sintesis
2000 .

Registro de la propiedod inte- depósito eue marca la les. IMPRESO EN LA ARGEN. TINA. Los articulos firmados que reflejan necesariamente is opi. nión de la revista.

COMPOSICION tipegratica en frío: TYFE S.R.L. Flarids
$253-40 \mathrm{M}$

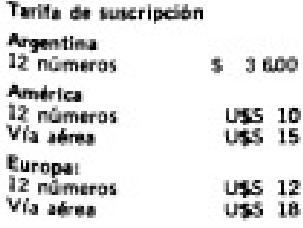

Cheques y giros a la orden de LOS LIBROS. Tecumin 1427, 2o. piso, of. 207 , Buenos $A$ i res, Argentina.
\end{abstract}

\section{Sumario}

Córdoba: la movilización permanente, noe

Francigco J. Delich

Guia Bibliograffiea

Les nueves sindicates, $D D$

Osvaldo Reica

DOCUMENTOS

Ei persamiento de SITRAC

EI Progroma de SITRAC-SITRAM

El movimiento estudiantil: de la Reforma al Cordobazo, por

Famón Quevas y Osvalda Reica

La acciàn guerrillera, por

Germán Aose

Medios de comunicación: ef lengaje y la pelitica, par

Manueis Montes y Sikina Fawion

Córdoba y la revolución social ista en la Arg̨entina, our

James Petras

Una opotieión sociald, por

Juan Carlos Torre

\section{En este número}

\begin{abstract}
El presente nümero de Las Libros adquiere singular importancia. Vinculada a Editorial Galerna desde su aparición, en adelante la revista funcionará con absoluta independencia. Resulta agradable valorar, justamente ahora, el esfuerzo realizado por una de las editoriales más jóvenes de la Argentina que hizo posible, no sin dificultades, el hecho infrecuente de asegurar la subsistencia de una publicación corno ésta. Gracias a esta circunstancia, gracias a los dos años de aparición corsecutiva, a la difusión alcan. zada en toda América Latina y al apoyo creciente de numerosas editoriales, Las Libros puede hoy asumir la responsabilidad de proseguir su salida regular con recursos propios.
\end{abstract}

Ante cada etapa, es útil reconocer lo andada La apuesta que habiamos hecho en un comienzo estabs cargada de riesgos. El subdesarrollo y la dependencia se insinúan en todas partes y por los más sutiles conductos. En nuestros paises no resulta fácil soste: ner la aparición de una revista que intenta mantenerse ajena a las coherciones del mercado. $Y$ no sólo por razones económicas. El hábito de traducir que ha presidido el ordenamiento de nuestra cultura, modelo una manera de pensar a la vez que limitaba el esfuerzo productivo de nuestros intelectuales. La experiencis de hacer una revista donde se meditara -a través de la crítica de libros- sobre los diversos aspectos que adquiere el pensar humano, mostró la unidireccionalidad de nuestra formación: más de una vez, numerosos temas dejaron de considerarse en las páginas de la revista porque no se encontró la persona adecuada para un adecuado tratamiento. Por otra parte, nos negamos sistemáticamente a ro: petir comentarios meramente descriptivos o valora. ciones cargadas de adjetivos. El reconocimiento de los limites con que nos enfrentábamos y las razones sociales que los determinaban, fue el primer resultado provechoso que nos dejó is práctica en que nos habiamos empeñado.

Con el tiempo, y a partir de las enserianzas que adquirimos, la revista modificó sus contenidos. El campo de preocupaciones se fue ampliando $y$ dejó de articular su existencia en función ủnica a la critica de libros. En el dialogo con colaboradores y lectores fue redefiniendo su sentido. Hoy, Las Li. bros apetece constituir un espacio adecuado para una critica politica de la cultura, lo que no significa abandonar las primeras propuestas. Por el contrario, se trata ahora de leer Con lucidez no sólo los textos que ofrece la escritura (cualquiera sea su caracteris. tica) sino también esos otros textos que constituven los hechos históricos sociales. Estas formas de la cultura exigen también una lectura que los decodifique para destacar su significado, el mundo ideolo. gico en que se insertan. Requieren ser ordenados a partir de un pensamiento que los observe como sintomas de una estructura que se ofrece opsca $y$ que es preciso develar para modificarla, en la medida que ha mostrado su naturaleza esencialmente opresora. Se trata, en ultima instancia, de contribuir al cambio de las condiciones en que se produce la cultura $y$ que incluye la posibilidad de una lectura radicalmente distinta de los libros. 

lector: ahora más que nunca depende de Ud.

A partir de este nimero, Los Libros fumciona con total autonomia económica. Sus dos atlos de existencia, la acepración que ba recibido tamto en la Argentina como en otros parises de América latima, le permiten mantener su aparicidn regalar sin el apoyo que basta abora le brindara la Editorial Galema

Abara, mads que wunca, la revista depende de sus amigos. Afrowtar el despegue de esta nuesa etapa requiere, por lo tanto, de sa apoyo. Suscribase pagando una tarifa espercial de apoyo. Si Ud. abowa 5100 (o 25 dolares para el exterior), ademads de los 12 mumeros comespondientes a su suscripcion por un alo, le exviaremes como obsequio wna celeccibin de los 20 mimeros aparecidos basta bey.

-

Srez. LOS UBROS

Tucumin 1427

Buenes Aires

Sirvawse suscribirme por un aี้อ (12 múmeros)

a la revisia LOS LIBROS

a partir del número. . . inclusine

Queda entendido que recibine sin cargo

una calección completa de la resista.

desile $\mathrm{dN}^{\circ} \mathrm{I}$ al 20. Adjunto cbeque

giro por valor de $\$ 100$

NOMBRE:

Dомісіно

GUDAD Y PAIS:

Cheques o giros a la orden de "LOS UBros"

Tucumin 1427, Buenos Aires.
DOU JUAN CARLOS TORRE

4 renuilt colectiog qat estalla en Condotey en mave \& 1969 on uny cardoca tis mayo of 1969 as una reacción contra una coyantura en 4 . que se combina un proyecto de racionelización económica capitalista y un poder fundado en el autoritarismo una oposición social.

Ubicado en el encuentro entre Io crisis de una sociedad urtana e industrial y la afirmación de una vociedad capitalisa, el cordobazo no podía evcapar a ia dialéctica que podia escapar a la diáćctica que anima el surgimiente de los movimientos sociales: vu practica se pro yecté mas alli de sus ideas. Mejor Sicho, en Cóndeba, la disociación cae se produce entre los comperta. mieneos y la representación que se dan de eflor les protagonistas de una transición on las luchas sociales * munifessó dramiticamente en 1 explositin de unu violencia in con ugas. Porgat is violencie on es cordobare no wibe so una resour. 4 a un poder actaritario $y$ un echazo a la previón como arma del combate social. Su estallido re vela al mismo tiempo el drama de una oposición social que aparece en el momento en que los tersione: provocadas por las desigusidades sociales y los obsticulos a la particinación polftica son desplanadas por lo chon politica son desplazadas por hos conitos desencadenados dindedor de una lucha de clases, Negindose nombrar el nuevo eccenario en e. que se instalsos la practica politic. con les simbolos del pasado, pero careciendo todavia de un Sxcurvo propio que ilumirara la originalidad wet introducian en lis luchas soxis. lex. los actores del cordebaro llens. rea eve vacio tierdiendo une violen. cho ondinimy y vintud?

Movimiento espresiog, on ave Morimiento expresivo, nn all are ento domina sobre la pelabra.

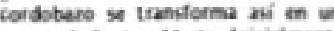
aconiecimitinto sobienta. Inicizimente \& ha imientado despejar su significa. do no viendo en a mas que ta prolongeción directe de los movi mientos populares contra la core. siôn sociall y la posteración econt. ricis. De este modo, el condeharo mica. De este modn, el cordoto se conviche en un capitio de antaganismo cpico entre dominantes $y$ dominados $y$ sus protagonislas 5 confunden con los montoneres y los descamisados en la reivindicación de la libertad y la justicia. Pero la exaltacion de un mismo espiritu revolucionario no detbe ocultar is mosificación operada en los acteres, en los cbietives y los conficiction.

L mevilización cbrera gae suced

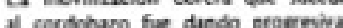

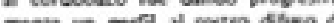

\section{Amigo \\ UNA NUEVA OPOSICION SOCIAL}

la revuelia y un programa a su discurbo ausente. El resumen de esta creación sedectiva hoy se encuentra creacion selesina hoy se encuentra representado en SITRCLSITRAM Y edefine los terminos clasicos dentro de los que se ha planteado la lucha social en la Arsenting. Frente a una histeria serita en nombre del pue. do por la conquista de la justicia secial y la participación política, los sindicatos "dwias" uargides del cordctous intentan iraururar otra,

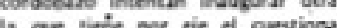

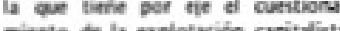
minto or la explotación capitalista Ya coneral etrero wobre el desarto. ino social. is a partir de este cam. bio en la identidad de lon actores y en los principios que animan su lucha gat $x$ ha propuesio, mis. teciememenie, un interpretación que comider a cordobuso ra no una continuidad sino el signo de una verdadera noptura.

Pere, sin ton elle res resupera

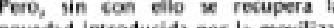
movedidinirodicids per la moviliza cion corera, seria, win embargo, erróneo entender a eta ruptura co mo el despertar de una concienci. de clase siempre in nuce. La con ciencia anti capicilista mo es un atri. bute inmanente a la condición abre a. oue puede ser sofuade por los dearatos burecratices o las iseolo

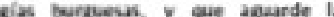

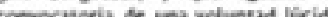

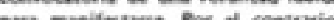

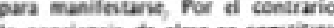

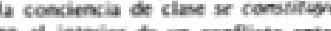
on ef imerior of un conflicto entre daves, es decir, un conflicto on of que los obreres enlremian a un adversarie vobre al teriene organize. do por la presencia de un poder capitaliata. En términos más genera les, la consiencie e trovers de lg cie se articido un movimiente saxigl no ave de definire inclupendiontemente

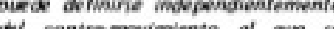
ati caniromovimiento a gue se SITRACSITRAM so deste SITRACSITRAM von, desde est perspectiva la respuesia del mundo del tratajo a la racionalización capiculiva que Krieger Vasena consagra. a come pelitica esonómica de Revolucion Argentina.

Que et dnueroillo del capitalismo en 4 seciedad iscention ha aleano 4. valo wancido si indiscutiele:

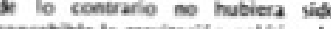
cencabiole la graickión politica de: proyecto woondmice prepuesto por Kritger Vave y adminisorado por cus continuadares vergonravites. Pe ro wis povibilidades pera hegemoni. zar la condocción del Estado $y$. su capacided para provocar una ruptura en las luchas saxieles peneralizand las condiciones de un conflicto claves, tes abn limitades. $L$ a avente

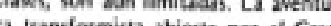
acuento Nasional a la por el Gra Acuerdo Nacional a la vec que indica que el proceso social iniciado en 1945 ague sin resolverse, marca el tiempo historike dentro del yue se inserta I lucha de ISTRACSITRAM. La nuera epenición wocial surgide del cordobo to antikipa un porvenir, ne es pode vis el centro de la historia pelitica inmediata. $\bullet$ 


\section{UNA NUEVA OPOSICIÓN SOCIAL POR JUAN CARLOS TORRE}

La revuelta colectiva que estalla en Córdoba en mayo de 1969 es una reacción contra una coyuntura en la que se combina un proyecto de racionalización económica capitalista y un poder fundado en el autoritarismo político y seala el nacimiento de una oposición social.

Ubicado en el encuentro entre la crisis de una sociedad urbana e industrial y la afirmación de una sociedad capitalista, el cordobazo no podía escapar a la dialéctica que anima el surgimiento de los movimientos sociales: su práctica se proyectó más allá de sus ideas. Mejor dicho, en Córdoba, la disociación que se produce entre los comportamientos y la representación que se dan de ellos los protagonistas de una transición en las luchas sociales se manifestó dramáticamente en la explosión de una violencia sin consignas. Porque la violencia en el cordobazo no sólo es una respuesta a un poder autoritario y un rechazo a la presión como arma del combate social. Su estallido revela al mismo tiempo el drama de una oposición social que aparece en el momento en que las tensiones provocadas por las desigualdades sociales y los obstáculos a la participación política son desplazadas por los conflictos desencadenados alrededor de una lucha de clases. Negándose a nombrar el nuevo escenario en el que se instalaba la práctica política con los símbolos del pasado, pero careciendo todavía de un discurso propio que iluminara la originalidad que introducían en las luchas sociales, los actores del cordobazo llenaron ese vacío ejerciendo una violencia anónima y virtual.

Movimiento expresivo, en el que el gesto domina sobre la palabra, el cordobazo se transforma así en un acontecimiento abierto. Inicialmente se ha intentado despejar su significado no viendo en él más que la prolongación directa de los movimientos populares contra la opresión social y la postergación económica. De este modo, el cordobazo se convierte en un capítulo del antagonismo épico entre dominantes y dominados y sus protagonistas se confunden con los montoneros y los descamisados en la reivindicación de la libertad y de la justicia. Pero la exaltación de un mismo espíritu revolucionario no debe ocultar la modificación operada en los actores, en los objetivos y los conflictos.

La movilización obrera que sucede al cordobazo fue dando progresivamente un perfil al rostro difuso de la revuelta y un programa a su discurso ausente. El resumen de esta creación colectiva hoy se encuentra representado en SITRAC-SITRAM y redefine los términos clásicos dentro de los que se ha planteado a lucha social en la Argentina. Frente a una historia escrita en nombre del pueblo por la conquista de la justicia social y la participación política, los sindicatos "clasistas" surgidos del cordobazo intentan inaugurar otra, la que tiene por eje el cuestionamiento de la explotación capitalista y el control obrero sobre el desarrollo social. Es a partir de este cambio en la identidad de los actores y en los principios que animan su lucha que se ha propuesto, más recientemente, una interpretación que considera al cordobazo ya no una continuidad sino el signo de una verdadera ruptura.

Pero, sin con ello ${ }^{3}$ se recupera la novedad introducida por la movilización obrera, sería, sin embargo, erróneo entender a esta ruptura como el despertar de una conciencia de clase siempre in nuce. La conciencia anti-capitalista no es un atributo inmanente a la condición obrera, que puede ser sofocado por los aparatos burocráticos o las ideologías burguesas, y que aguarde la convocatoria de una voluntad lúcida para manifestarse. Por el contrario, la conciencia de clase se constituye en el interior de un conflicto entre clases, es decir, un conflicto en el que los obreros enfrentan a un adversario sobre el terreno organizado por la presencia de un poder capitalista. En términos más generales, la conciencia a través de la cual se articula un movimiento social no puede definirse independientemente del contra-movimiento al que se opone. Las consignas socialistas de SITRAC-SITRAM son, desde esta perspectiva, la respuesta del mundo del trabajo a la racionalización capitalista que Krieger Vasena consagrara como política económica de la Revolución Argentina. 
Que el desarrollo del capitalismo en la sociedad argentina ha alcanzado un grado avanzado es indiscutible: de lo contrario no hubiera sido concebible la gravitación política del proyecto económico propuesto por Krieger Vasena y administrado por sus continuadores vergonzantes. Pero sus posibilidades para hegemonizar la conducción del Estado y su capacidad para provocar una ruptura en las luchas sociales generalizando las condiciones de un conflicto de clases, son aún limitadas. La aventura transformista abierta por el Gran Acuerdo Nacional a la vez que indica que el proceso social iniciado en 1945 sigue sin resolverse, marca el tiempo histórico dentro del que se inserta la lucha de SITRAC-SITRAM. La nueva oposición social surgida del cordobazo anticipa un porvenir, no es todavía el centro de la historia política inmediata.

\section{Notas}

1 Es posible acceder a todos los ejemplares completos de Los Libros a través del portal América Lee organizado por el CEDINCI: http://americalee.cedinci.org/portfolio-items/los-libros/

2 Destacamos especialmente su artículo "A partir del Cordobazo", publicado en Estudios no 4, 1994, a raíz del 25 aniversario de la gesta cordobesa.

3 Nota del propio autor para la presente reproducción: donde dice "Pero sin con ello", debería decir "Pero si con ello". 